\title{
Site-Controlled VLS Growth of Planar Nanowires: Yield and Mechanism
}

\author{
3 Chen Zhang, Xin Miao, Parsian K. Mohseni, Wonsik Choi, and Xiuling Li* \\ 4 Department of Electrical and Computer Engineering, Micro and Nanotechnology Laboratory, University of Illinois at \\ 5 Urbana-Champaign, Urbana, Illinois 61801, United States
}

6

Supporting Information

\begin{abstract}
The recently emerged selective lateral epitaxy of semiconductor planar nanowires (NWs) via the vaporliquid-solid (VLS) mechanism has redefined the longstanding symbolic image of VLS NW growth-vertical NWs extending out of the substrate. The in-plane geometry and selfaligned nature make these planar NWs completely compatible with large scale manufacturing of NW-based integrated nanoelectronics. Here, we report on the realization of perfectly site-controlled growth of GaAs planar NW arrays with unity
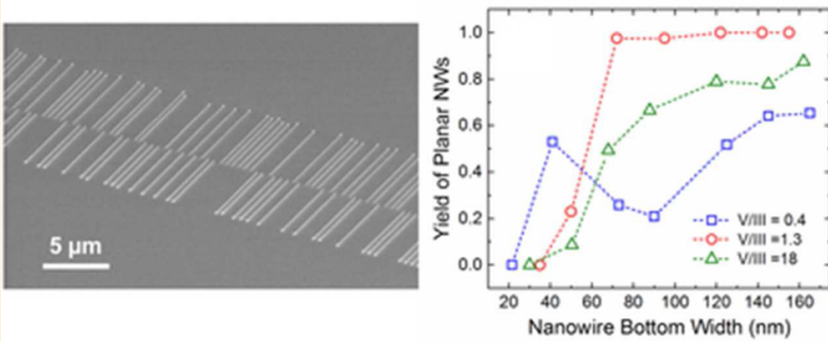
yield using lithographically defined gold $(\mathrm{Au})$ seed dots. The growth rate of the planar NWs is found to decrease with the NW width when spacings are fixed, which is consistent with the conventional model where the Gibbs-Thomson effect is considered. It is found that in general, the planar and out-of-plane NW growth modes are both present. The yield of planar NWs decreases as their lateral dimension shrinks, and $100 \%$ yield of planar NWs can be achieved at moderate V/III ratio. Based on a study of the shape of seed particles, it is proposed that the adhesion between the liquid-phase seed particle and the substrate surface is important in determining the choice of growth mode. These studies represent advances in the fundamental understanding of the VLS planar NW growth mechanism and in the precise control of the planar NW site, density, width, and length for practical applications. In addition, high quality planar InAs NWs on GaAs (100) substrates is realized, verifying that the planar VLS growth mode can be extended to heteroepitaxy.

KEYWORDS: Vapor-Liquid-Solid Mechanism, Planar Nanowire, MOCVD, GaAs, InAs
\end{abstract}

26 emiconductor nanowires (NWs) hold extreme promises to ${ }_{27} \circlearrowleft$ be the building blocks of future electronics and photonics 28 and, thus, have received tremendous attention for many 29 years. $^{1-7}$ Among all kinds of fabrication methods, the 30 bottom-up vapor-liquid-solid (VLS) growth method, ${ }^{8}$ where 31 a metallic (e.g., Au) seed particle is used to gather materials and 32 guide NW growth, attracts particular interests owing to its 33 advantages in size downscaling, versatility in the incorporation 34 of doping and heterostrutures, ${ }^{9-12}$ and potential in heteroge35 neous NW integration on foreign substrate ${ }^{13-17}$ (for example, $36 \mathrm{III}-\mathrm{V}$ NWs on silicon). Ever since the VLS method was 37 invented, ${ }^{8}$ NWs standing on substrates and pointing out along 38 surface normal directions have served as the symbolic picture of 39 this method. For III-V materials, these out-of-plane NWs 40 mostly grow along $\langle 111\rangle \mathrm{B}$ direction. ${ }^{18}$ The out-of-plane 41 geometry is not compatible with the well-established planar 42 processing technologies for field-effect transistors (FETs) and, 43 therefore, requires additional processing effort for device 44 integration. More importantly, the vertical channel structure 45 tends to incur more parasitic capacitance, which degrades the 46 frequency performance (speed) of the device. So far, vertical 47 NW FETs have not shown advantages in speed in comparison 48 to the planar thin-film FETs given the same channel 49 material. ${ }^{19-21}$ In this light, selective lateral epitaxy of planar $50 \mathrm{NW}$ growth method, ${ }^{22,23}$ where NWs are grown out of Au seed particles, via the VLS mechanism, propagating laterally along 51 certain in-plane directions in a self-aligned fashion, has recently 52 emerged. This growth method has not only enriched the 53 fundamental VLS study but also provided a practically useful 54 technology for realizing NW-based integrated circuits. So far 55 high-performance MESFETs, HEMTs, and MOSFETs devices 56 have been demonstrated using planar NWs as the current 57 conducting channels. ${ }^{24-27}$ Circuits have also been demon- 58 strated by interconnecting aligned planar NW MESFET 59 devices. $^{28}$ To advance the fundamental VLS study and to 60 better assess the technological potential, a systematic growth 61 study is needed. For doing so, we have first developed array- 62 based GaAs planar NW growth using electron beam 63 lithography (EBL) to predefine the Au seed particles. 64

Figure $1 \mathrm{a}$ and $\mathrm{b}$ show representative scanning electron $65 \mathrm{fl}$ microscope (SEM) images of a GaAs planar NW array grown 66 on GaAs (100) substrate. The patterned Au dot array was 67 placed at a horizontal line as indicated by red arrows. The 68 center to center spacing between the NWs is $1 \mu \mathrm{m}$. The sample 69 shown here was grown in a metal-organic chemical vapor 70 deposition (MOCVD) system under 950 mbar at temperature 71

Received: July 5, 2014

Revised: October 8, 2014 

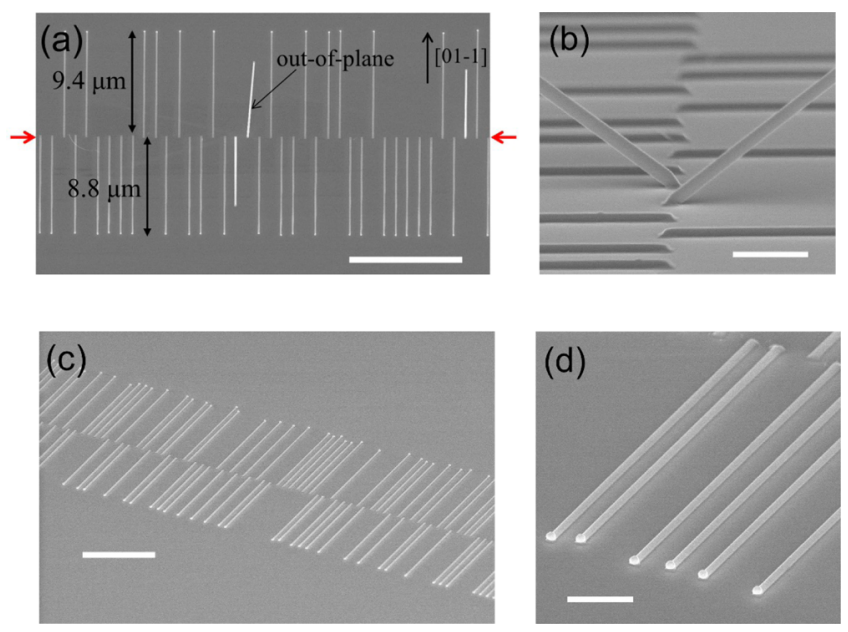

Figure 1. SEM images of GaAs planar NW arrays grown on GaAs (100) substrates. (a) Top-view SEM image of a representative GaAs planar NW array on a (100) substrate. The red arrow indicates where the line of Au dots was initially patterned. Planar NWs were formed as the Au dots propagated upward $\left(\left[\begin{array}{lll}0 & 1 & -1\end{array}\right]\right.$ direction) or downward $\left(\left[\begin{array}{ll}0 \\ 0\end{array}\right.\right.$ $-11])$ during the growth. The three brighter NWs are out-of-plane as indicated. The NW bottom width (trapezoidal cross-section) of this particular array is $145 \mathrm{~nm}$. (b) An $80^{\circ}$ tilted-view SEM image of the same sample in (a) clearly showing two out-of-plane NWs. (c) A $60^{\circ}$ tilted view of a planar NW array with perfect yield of planar NWs achieved by optimizing the growth condition (see text for details). (d) Higher magnification view of the same array in (c). The Au catalyst dots, which propagated from the center line to guide the NW growth, are clearly visible at the tips of those NWs. The scale bars are 10, 1, 5, and $1 \mu \mathrm{m}$ for $(\mathrm{a})-(\mathrm{d})$, respectively.

$72 \mathrm{~T}=460{ }^{\circ} \mathrm{C}$ for $80 \mathrm{~s}$ with a nominal V/III ratio of 30 . The 73 bidirectional growth ${ }^{22,29}$ can be clearly seen in Figure 1a. 74 Interestingly, we observe a noticeable difference in the planar $75 \mathrm{NW}$ lengths thus growth rates between the two presumably 76 crystallographically equivalent directions, with the NWs 77 propagating along $\left[\begin{array}{lll}0 & 1 & -1\end{array}\right]$ and the antiparallel $\left[\begin{array}{lll}0 & -1 & 1\end{array}\right]$ 78 direction being 9.4 and $8.8 \mu \mathrm{m}$ in length, respectively. Such 79 growth rate difference (typically $<20 \%$ ) is only observed on 80 large diameter NWs and disappears when the NW widths are 81 smaller than $\sim 80 \mathrm{~nm}$. This phenomenon is not the major point 82 here and needs further investigation. We speculate that the 83 preference could be induced by substrate orientation miscut 84 (manufacture specification was $\pm 0.5^{\circ}$ ), which makes the two 85 directions no longer perfectly equivalent. On this particular 86 sample, a few out-of-plane NWs are observed and can be 87 identified as those brighter (and shorter) NWs in Figure 1a. 88 The out-of-plane NWs grow along $\langle 111\rangle$ B directions and, 89 therefore, are aligned at $35.3^{\circ}$ with respect to the substrate 90 surface. $^{22}$ Figure $1 \mathrm{~b}$ is a tilted-view SEM image where two out91 of-plane NWs can be clearly seen. Remarkably, the yield of the 92 planar NWs can be improved by tuning the growth conditions 93 (details will be discussed later). Figure $1 \mathrm{c}$ and $\mathrm{d}$ show 94 exemplary SEM images of the planar NW array with unity yield. 95 A comparison between the growth rates of planar and out-of96 plane NWs is shown in Figure 2. For out-of-plane NWs, the 97 growth direction (direction of facet advancing) and the normal 98 direction of growth front are the same (both $[111] \mathrm{B}){ }^{18}$ as 99 illustrated by the top schematic in Figure 2a. However, the two 100 directions are different for the planar NW growth. As shown in 101 Figure 2a (bottom schematic), the angle $\theta$ between the two 102 directions is $35.3^{\circ}$ for planar NW growth on GaAs (100)
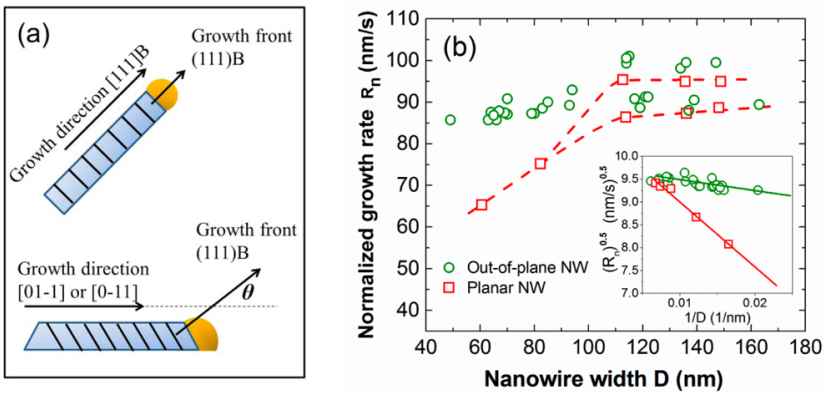

Figure 2. Comparison between growth rates of planar and out-ofplane GaAs NWs. (a) Schematic diagrams of out-of-plane and planar NWs grown on (100) substrate. A normalized growth rate is introduced in the main text for a fair comparison of the two growth modes. (b) Comparison between normalized growth rates of planar NWs and nonplanar NWs. The growth was done at $460{ }^{\circ} \mathrm{C}$ with a V/ III ratio of 30 under 950 mbar. Each data point (red squares) for planar NWs was measured and averaged from an array with $10 \mu \mathrm{m}$ spacing. The data for out-of-plane NWs were measured on the same arrays. Each data point (green circles) refers to the growth rate of an individual NW. The dashed lines are only for eye guidance. The inset of (b) shows a linear fit to the square root of growth rate versus the inverse of NW width for both growth modes.

substrates because the growth direction is either $\left[\begin{array}{lll}0 & 1 & -1\end{array}\right]$ or $\left[\begin{array}{ll}0 & 103\end{array}\right.$ $\left.\begin{array}{ll}-1 & 1\end{array}\right]$, whereas the growth front is still $(111) \mathrm{B}$, according to 104 our previous studies. ${ }^{22,29}$ Let $R_{\mathrm{m}}$ be the measured NW growth 105 rate, which can be obtained by directly dividing the measured 106 NW length by the growth time. The volume of material 107 deposited per unit area per unit time is $R_{\mathrm{m}} \cos \theta$, where $\theta$ is $0^{\circ}{ }_{108}$ for out-of-plane NWs and $35.3^{\circ}$ for planar ones. So the number 109 of atoms deposited per unit time per unit area is proportional 110 to $R_{\mathrm{m}} \cos \theta$. Therefore, we define a normalized growth rate as $R_{\mathrm{n}} 111$ $=R_{\mathrm{m}} \cos \theta$ in order to compare the two different types of NWs 112 on an equal footing.

Figure $2 \mathrm{~b}$ shows the normalized growth rates of planar NWs 114 and out-of-plane NWs measured from the sample prepared 115 under the same growth condition as in Figure 1a. Each of the 116 data points (red squares) for planar NWs was measured and 117 averaged from about 20 NWs in an array with $10 \mu \mathrm{m}$ spacing 118 between adjacent NWs. The large spacing was designed to 119 minimize any synergetic effect of growth rate between 120 neighboring NWs. ${ }^{30}$ Without spacing control in the growth 121 rate study, the real trend can be hidden by density related 122 variations. Five arrays of holes were first patterned on PMMA 123 by EBL with nominal diameters of 300, 250, 200, 150, and 100124 $\mathrm{nm}$, respectively. Au dots were then produced by a lift-off 125 process after $30 \mathrm{~nm}$ Au film was evaporated. The planar NWs 126 are very uniform in size and growth rate within each array. The 127 standard deviations of growth rate and width are smaller than 3128 $\mathrm{nm} / \mathrm{s}$ and $3 \mathrm{~nm}$, respectively. As seen from Figure 2b, when the 129 $\mathrm{NW}$ width is relatively large ( $>\sim 110 \mathrm{~nm}$ in bottom width), two 130 different growth rates are associated with each planar NW size. 131 The growth rate starts to roll off clearly when the width 132 becomes smaller. This is consistent with the conventional VLS 133 model proposed by Givargizov, ${ }^{31}$ where smaller NWs are 134 associated with slower growth rates because NW surface energy 135 reduces supersaturation. The inset of Figure $2 \mathrm{~b}$ plots the square 136 root of growth rate versus the inverse of NW width (the data 137 points with slower growth rate are adopted for the bimodal 138 cases). A fairly good linear fit can be obtained for planar NWs, 139 supporting the interpretation by the conventional model. ${ }^{31,32} 140$ 

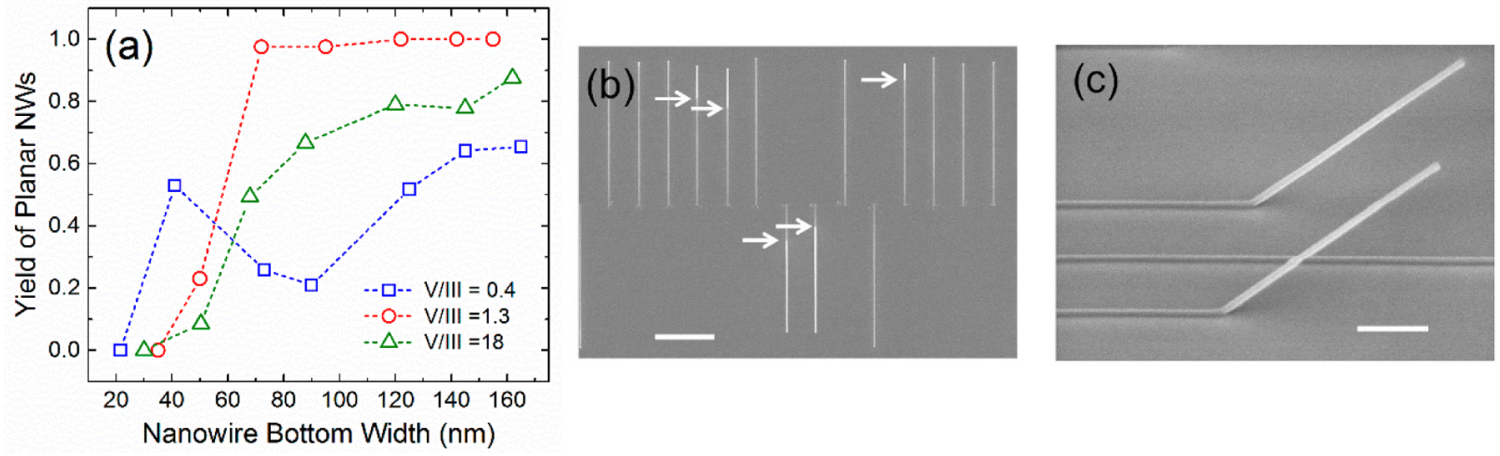

Figure 3. Yield study of VLS GaAs planar NWs. (a) Yield of planar NWs as a function of bottom width. Three samples were prepared with V/III ratios of $0.4,1.3$, and 18 , respectively. To vary V/III ratio, we fixed TMGa flow and only the $\mathrm{AsH}_{3}$ flow was changed across the three samples. Other growth conditions were identical for all the three samples. The growth temperature and reactor pressure were $460{ }^{\circ} \mathrm{C}$ and $150 \mathrm{mbar}$, respectively. (b) Typical SEM image showing the transition regime where some of the planar NWs start to take off from the substrate surface in the middle of the growth. The out-of-plane NWs show brighter contrast in the image. The white arrows indicate the points where the NWs start to take off. The scale bar is $2 \mu \mathrm{m}$. (c) A $60^{\circ}$ tilted view of the NWs taking off in the middle of the growth. The scale bar is $500 \mathrm{~nm}$.

141 By extrapolating the fitted line, the critical width is calculated to 142 be $\sim 13 \mathrm{~nm}$ for the planar growth.

143 We were able to measure the length of out-of-plane NWs 144 also from those arrays because the yield of planar NWs was not 145 perfect under that particular growth condition. Note that each 146 of the data points (green circles) of out-of-plane NWs only 147 refers to the growth rate measured from an individual NW. The 148 bimodal rate is also seen for the out-of-plane NWs. The 149 normalized growth rates of planar NWs (slightly slower) match 150 closely with that of out-of-plane NWs, both of which remain a 151 near constant value. This can be understood by the fact that the 152 Gibbs-Thomson effect ${ }^{31}$ (NW sidewall energy) is weak for 153 thick NWs and thus out-of-plane and planar NWs should have 154 very supersaturation, $\Delta \mu$, defined as the difference between 155 chemical potentials of NW materials in the vapor phase and in 156 the NW. ${ }^{31}$ As the NW width shrinks down, the growth rate of 157 planar NWs decreases steeper than that of out-plane NWs. 158 Note that this trend, where the two modes have close growth 159 rates at large sizes but planar NW grows slower at smaller sizes, 160 is true for all growth conditions we have tested. This implies 161 that for smaller sizes, out-of-plane growth is associated with 162 higher supersaturation. In the discussion above, we assume the 163 kinetic parameters that connect supersaturation to growth rate 164 are the same for both types of growth. Though it is open for 165 discussion, it is reasonable because both planar and out-of-plane 166 NWs are seeded by Au and have (111)B growth front. Because 167 they are located on the same sample, they also had experienced 168 exactly identical growth conditions. Another factor that needs 169 to be considered is the surface adatom diffusion, which can 170 affect the NW growth rate and cannot be captured by the 171 conventional model. ${ }^{33}$ We have confirmed that the NW growth 172 rate induced by surface diffusion is negligibly small in our 173 experiment (below $1 \mathrm{~nm} / \mathrm{s}$, see Supporting Information for the 174 calculation). Note that our observation here is different from an 175 insightful previous study on InAs planar NWs, ${ }^{34}$ where it was 176 shown that planar growth was associated with larger super177 saturation (less suffered from Gibbs-Thomson effect) due to 178 the removal of substrate surface by planar growth. The 179 contradiction is worth further study, and we speculate that 180 under our growth conditions, the top (100) and sidewall 181 (111)A surface energy of the planar NW is large and makes the 182 overall supersaturation of planar growth smaller despite the 183 removal of the bottom surface.
Figure 3a shows the yield of planar NWs as a function of the $184 \mathrm{f3}$ NW size (bottom width). The yield here is defined as the ratio 185 between the number of NWs that remain planar throughout the 186 entire growth period and the total number of NWs (81) grown 187 in the array. Three samples grown with different nominal V/III 188 molar flow ratios (0.4, 1.3 and 18) are shown in Figure 3a. To 189 vary V/III ratios, the TMGa flow was fixed and only the $\mathrm{AsH}_{3} 190$ flow was changed across the three samples. Other growth 191 conditions were identical, with growth temperature and reactor 192 pressure being $460{ }^{\circ} \mathrm{C}$ and 150 mbar, respectively. Multiple Au 193 dot arrays with the same dot size within each array were 194 patterned on each of those samples. The growth rates of large 195 NWs (>120 nm in width) on the samples with V/III ratio of 196 $18,1.3$, and 0.4 are 135,120 , and $40 \mathrm{~nm} / \mathrm{s}$, respectively. The 197 corresponding growth times are 50,50, and $80 \mathrm{~s}$, respectively. 198 For smaller NWs, the growth rate decreases following similar 199 trend shown in Figure 2b. From Figure 3a, it can be seen that 200 for NWs with bottom width larger than $\sim 70 \mathrm{~nm}$, the sample 201 with $\mathrm{V} / \mathrm{III}=1.3$ has unity yield of planar NWs. The yields from 202 the other two samples are noticeably lower. In this size range, 203 unity yield can be reproducibly achieved when the V/III ratio 204 ranges from $\sim 0.8$ to $\sim 5$.

As the NW width becomes smaller, the yield at the optimum 206 V/III ratio of 1.3 drops below $100 \%$, and when the width 207 becomes less than $\sim 50 \mathrm{~nm}$, no planar NWs can be observed. 208 The widths shown in Figure 3a for the zero yield case were 209 measured on out-of-plane NWs. Shown in Figure 3b is a typical 210 SEM image of the transition regime where the planar NW yield 211 has degraded. It is observed that many NWs start the growth in 212 the planar mode but take off from the substrate surface at some 213 point, as indicated by the white arrows in Figure 3b, during the 214 growth. This can be seen more clearly from Figure $3 \mathrm{~b}$. The 215 result suggests the presence of a delicate balance between 216 planar and out-of-plane mode. The other two samples show a 217 similar trend in general, where smaller NWs have lower yield 218 and no planar NWs can be found when the size drops below 219 certain points. A hump at $40 \mathrm{~nm} \mathrm{NW}$ width is observed on the 220 yield-size curve with $\mathrm{V} / \mathrm{III}=0.4$, which is not understood at 221 this point and needs further study. It has to be mentioned that 222 the cessation of planar NW growth at very small sizes is not 223 because of a complete loss of supersaturation due to the 224 Gibbs-Thomson effect. Extremely high growth rate $(\sim 100225$ $\mathrm{nm} / \mathrm{s}$ ) for planar NWs are still observed from the very low-yield 226 array of small-size NWs. In other words, there still exists a large 227 
228 level of supersaturation to support fast planar NW growth. 229 Other underlying reasons that control the preference between 230 planar and nonplanar growth must be considered.

231 Shown in Figure 4 are two SEM images of $\mathrm{Au}$ seeds after 232 growth, where $4 \mathrm{a}$ and $\mathrm{b}$ show the samples that were cooled
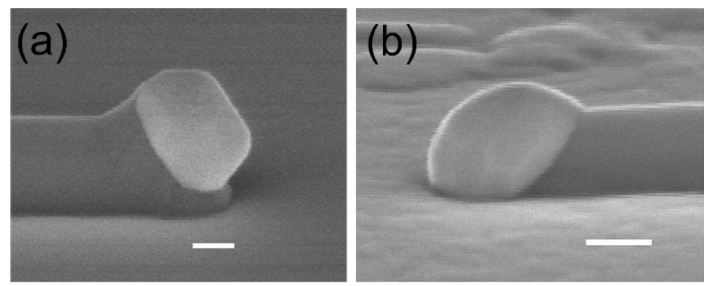

Figure 4. SEM images showing the shapes of seed particles after sample cooling. (a) Cooling with $\mathrm{AsH}_{3}$ overpressure. (b) Cooling without $\mathrm{AsH}_{3}$ overpressure. The sample is tilted by $80^{\circ}$. The scale bars are both $100 \mathrm{~nm}$.

233 down from the growth temperature with and without $\mathrm{AsH}_{3}$ 234 overpressure, respectively. It is observed that the $\mathrm{Au}$ particle in 235 Figure 4a shows a nonspherical shape and a neck-like structure 236 is formed and clearly visible below the Au nanoparticle. This is 237 because with the supply of As precursor during sample cooling, 238 the $\mathrm{Ga}$ atoms in the seed particle can continue to precipitate 239 out to form an additional growth segment of GaAs, as is the 240 case for out-of-plane NWs. ${ }^{35}$ However, the $\mathrm{Au}$ particle in 241 Figure 4b exhibits a rounder profile and only negligible 242 extraneous material is visible below the gold. In the absence of $243 \mathrm{AsH}_{3}$ supply, only a very small amount of GaAs can be formed 244 during cooling process because of the extremely low solubility 245 of $\mathrm{As}$ in $\mathrm{Au}$, so the shape of catalyst can be well preserved. 246 Therefore, Figure $4 \mathrm{~b}$ should more closely represent the actual 247 growth-phase geometry of $\mathrm{Au}$ nanoparticle. Importantly, the 248 base of the nanoparticle is in contact with the substrate, which 249 suggests that it was in direct contact with (wetted) the 250 substrate, in addition to the NW growth front, during planar $251 \mathrm{NW}$ growth. Note that in the growth of in-plane InAs NWs, 252 such additional contact interface was also observed. ${ }^{23,34}$

253 Based on the experimental analysis above, we propose that 254 the wetting nature of seed droplet on the substrate during 255 growth is an important factor responsible for the planar type of 256 VLS GaAs NW growth. Once the liquid-form seed particle 257 contacts the substrate surface, it needs to overcome the 258 adhesion energy between the seed and the substrate surface 259 before the out-of-plane mode may proceed. Note that it was 260 suggested, in the case of in-planar InAs traces growth on (111) $261 \mathrm{~B} \mathrm{GaAs},{ }^{23}$ that the $\mathrm{Au}$ catalyst stays on the substrate because $262 \mathrm{Au} / \mathrm{GaAs}$ interfacial energy is lower than that of $\mathrm{Au} / \mathrm{InAs}$ 263 interface. We believe that in general, this energy difference is 264 not necessary for the occurrence of planar VLS NW growth 265 because in our case, the $\mathrm{Au} / \mathrm{GaAs}$ (111)B interface at the 266 growth front should have lower interfacial energy. ${ }^{18} \mathrm{We}$ 267 speculate that the wetting was initiated during oxide desorption 268 procedure at the very beginning of the growth-the gold dots 269 collect materials from the substrate and become eutectic 270 droplets. After the growth precursors are introduced, the planar 271 growth proceeds with materials stacking in a layer-by-layer 272 manner on (111)B facet while the droplet remains in contact 273 with the (100) substrate since (100) is not the growth front. As 274 discussed in refs 36 and 37, perturbations that occur during 275 NW growth might strongly affect the choice of growth modes. 276 Perturbations such as organic residue on the substrate or the occurrence of a stacking fault may interrupt the contact 277 between the seed particle and the substrate and make the NWs 278 switch to the out-of-plane mode. Supporting evidence can be 279 found in our previous study, where the intentional introduction 280 of twinning defects by dopant incorporation can cause the 281 planar NW to switch to out-of-plane growth mode. ${ }^{38}$ This 282 explains the fact that even the yield for large planar NWs is not 283 always perfect except for under optimized V/III ratios. As 284 reported in the recent research on in situ TEM observation of 285 III-V VLS NW growth, ${ }^{39}$ a growth condition with very high V/ 286 III ratio can induce twinning defects, which is likely to disturb 287 the planar growth in our case. The factors discussed above also 288 provide a natural explanation for the size-dependent yield 289 study. Small-size seeds would have less contact area with the 290 substrate so one would expect that it is easier to separate them 291 from the substrate. In other words, the growth of narrower 292 planar NWs should be more vulnerable to perturbations and, 293 thus, shows lower yield. The analysis here implies that the 294 planar type of growth could be universally achievable in any 295 material system if (i) the growth front is not in parallel with the 296 substrate surface and (ii) the adhesion between the substrate 297 and the catalyst can be engineered to be sufficient.

298

At the end, we demonstrate here that planar III-V NW 299 growth may be extended to the heteroepitaxy of self-aligned 300 and high-quality InAs on GaAs (100) substrates through the 301 same VLS approach defined above. Growth of planar InAs 302 NWs on a semi-insulating substrate can be a very competitive 303 platform technology for future low-power digital applications 304 given the extremely high electron mobility in InAs. Despite the 305 approximately $6.7 \%$ bulk lattice-mismatch, directly interfaced 306 and single-crystalline lateral growth of InAs NWs is realized 307 under optimized growth conditions, with NWs being self- 308 aligned along the $\langle 011\rangle$ direction on GaAs (100) substrates. 309 Figure 5a shows a tilted-view SEM image of two as-grown InAs $310 \mathrm{fs}$ NWs on GaAs (100). We note that Au-seed particles are clearly 311 identified at the NW tips, characteristic of VLS-type growth, 312 whereas the NW widths exhibits no structural variations 313 (twinning or tapering) along their lengths. Figure 5b shows a 314 cross-sectional view high-resolution transmission electron 315 microscopy (HR-TEM) image of a laterally grown InAs NW 316 on GaAs. Although the NW body and GaAs substrate share an 317 atomically abrupt interfacial plane (dashed line, Figure 5b), the 318 $\mathrm{Au}$ seed appears raised relative to the substrate surface, likely 319 resulting from postgrowth termination precipitation of growth 320 species from the liquid-phase alloy in an $\mathrm{AsH}_{3}$-rich environ- 321 ment. Analytic-TEM characterization (refer to Supporting 322 Information) confirms the heteroepitaxial NW crystal quality 323 (no stacking faults) and composition.

In summary, we have performed a systematic experimental 325 study of the VLS growth of GaAs planar NWs from various 326 aspects. The size-dependent growth rate and yield of planar 327 NWs have been studied. Careful control of growth conditions 328 can lead to unity yield of planar NWs. We have also shown that 329 the liquid-phase seed particle contacts the substrate surface 330 during growth and proposed that the adhesion energy is 331 important in determining the choice of growth mode. We 332 believe that the well-controlled planar type of VLS growth can 333 be extended beyond the scope of homoepitaxy and it is 334 demonstrated by the growth of high-quality (free of stacking 335 faults) InAs planar NWs on GaAs (100) substrates. 336

Methods. Planar NW Growth. To grow GaAs planar NW 337 arrays, we first defined Au seed particles on GaAs (100) 338 substrate by electron beam lithography patterning (with a Raith 339 

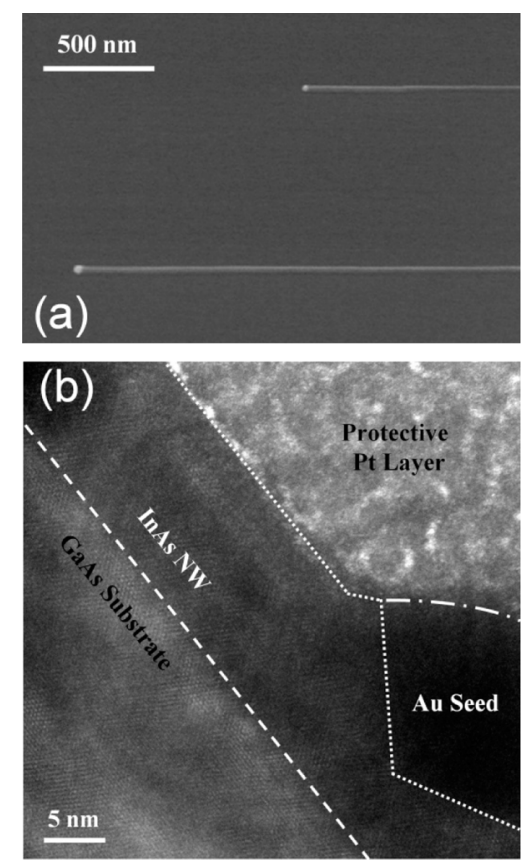

Figure 5. Heteroepitaxial VLS InAs planar NWs on semi-insulating GaAs (100). (a) Tilted-view SEM image showing parallel growth of two planar InAs NWs on a GaAs substrate. (b) Cross-sectional HRTEM image of a $\sim 12 \mathrm{~nm}$-thick planar InAs NW directly interfaced with the underlying GaAs substrate.

340 e-line system) and electron beam evaporation of Au. The 341 samples then went through an intensive cleaning procedure to 342 ensure the removal of organic residues. The samples were then 343 loaded into an Aixtron 200 atmospheric pressure MOCVD 344 reactor, followed by an oxide desorption step at $680{ }^{\circ} \mathrm{C}$. The 345 temperature was then lowered to $460{ }^{\circ} \mathrm{C}$ and GaAs NW arrays 346 were grown by using $\mathrm{AsH}_{3}$ and Trimethyl-gallium (TMGa) as 347 As and Ga precursors, respectively. The planar InAs NW 348 growth was carried out in the same reactor. Au nanoparticles (5 $349 \mathrm{~nm}$ in diameter) were randomly dispersed on the GaAs (100) 350 substrate from the commercial $\mathrm{Au}$ colloidal solution 351 (BBInternational, U.K.). After an oxide desorption step at $352625{ }^{\circ} \mathrm{C}$, InAs planar NWs were then grown at $360{ }^{\circ} \mathrm{C}$ with $353 \mathrm{AsH}_{3}$ and trimethylindium (TMIn) as precursors.

354 Characterization. The GaAs planar NW samples were 355 inspected by a Hitachi S4800 SEM instrument. The nanowire 356 length and bottom width were determined by the same SEM 357 instrument. A JEOL 2010F system was employed for all 358 scanning transmission electron microscopy (STEM) experi359 ments. Single NW TEM lamellae were prepared using an FEI 360 Helios NanoLab 600i focused ion-beam (FIB) system.

\section{ASSOCIATED CONTENT}

\section{S Supporting Information}

363 An estimation of growth rate contributed from surface 364 diffusion; A HR-TEM image obtained at the InAs NW/GaAs 365 substrate interface and corresponding FFT pattern; a HAADF366 STEM image showing the tip of a heteroepitaxial, planar InAs $367 \mathrm{NW}$; a figure showing the result of EDXS line-scan from the $368 \mathrm{GaAs}$ substrate to the NW body then to the Au seed particle, 369 which confirms the chemical composition of the InAs NW. 370 This material is available free of charge via the Internet at 371 http://pubs.acs.org.

\section{AUTHOR INFORMATION}

Corresponding Author

* E-mail: xiuling@illinois.edu.

Notes

The authors declare no competing financial interest.

\section{ACKNOWLEDGMENTS}

We are grateful for the support from the Division of Materials 378 Research (DMR) from NSF under grant \#1006581. Materials 379 growth experiments were carried out in the Micro and 380 Nanotechnology Laboratory, University of Illinois. P.K.M. 381 gratefully acknowledges FIB assistance from Dr. Jim Mabon. 382 Materials characterization experiments were carried out in part 383 in the Frederick Seitz Materials Research Laboratory Central 384 Research Facilities, University of Illinois.

\section{REFERENCES}

(1) Cui, Y.; Lieber, C. M. Science 2001, 291, 851-853. $241-245$.

(3) Xu, S.; Qin, Y.; Xu, C.; Wei, Y.; Yang, R.; Wang, Z. L. Nat. 390 Nanotechnol. 2010, 5, 366-373.

(4) Takei, K.; Takahashi, T.; Ho, J. C.; Ko, H.; Gillies, A. G.; Leu, P. 392 W.; Fearing, R. S.; Javey, A. Nat. Mater. 2010, 9, 821-826.

(5) Tomioka, K.; Yoshimura, M.; Fukui, T. Nature 2012, 488, 189- 394 192.

(6) Bertness, K. A.; Sanford, N. A.; Davydov, A. V. IEEE J. Sel. Topics 396 Quantum Electron. 2011, 17, 847-858.

(7) Nguyen, H. P. T.; Zhang, S.; Cui, K.; Han, X.; Fathololoumi, S.; 398 Couillard, M.; Botton, G. a; Mi, Z. Nano Lett. 2011, 11, 1919-1924. 399

(8) Wagner, R. S.; Ellis, W. C. Appl. Phys. Lett. 1964, 4, 89-90. 400

(9) Perea, D. E.; Hemesath, E. R.; Schwalbach, E. J.; Lensch-Falk, J. 401 L.; Voorhees, P. W.; Lauhon, L. J. Nat. Nanotechnol. 2009, 4, 315-319. 402

(10) Tian, B.; Zheng, X.; Kempa, T. J.; Fang, Y.; Yu, N.; Yu, G.; 403 Huang, J.; Lieber, C. M. Nature 2007, 449, 885-889.

(11) Lind, E.; Persson, A. I.; Samuelson, L.; Wernersson, L.-E. Nano 405 Lett. 2006, 6, 1842-1846.

(12) Qian, F.; Li, Y.; Gradecak, S.; Wang, D.; Barrelet, C. J.; Lieber, 407 C. M. Nano Lett. 2004, 4, 1975-1979. 408

(13) Mårtensson, T.; Svensson, C. P. T.; Wacaser, B. A.; Larsson, M. 409 W.; Seifert, W.; Deppert, K.; Gustafsson, A.; Wallenberg, L. R.; 410 Samuelson, L. Nano Lett. 2004, 4, 1987-1990.

(14) Davydok, A.; Breuer, S.; Biermanns, A.; Geelhaar, L.; Pietsch, U. 412 Nanoscale Res. Lett. 2012, 7, 109.

(15) Mazid Munshi, M.; Dheeraj, D. L.; Fauske, V. T.; Kim, D.-C.; 414 van Helvoort, A. T. J.; Fimland, B.-O.; Weman, H. Nano Lett. 2012, 415 $12,4570-4576$.

(16) Woodruff, J. H.; Ratchford, J. B.; Goldthorpe, I. a; McIntyre, P. 417 C.; Chidsey, C. E. D. Nano Lett. 2007, 7, 1637-1642. 418

(17) Mohseni, P.; Behnam, A.; Wood, J.; English, C. D.; Lyding, J. 419 W.; Pop, E.; Li, X. Nano Lett. 2013, 13, 1153-1161. 420

(18) Fortuna, S. A.; Li, X. Semicond. Sci. Technol. 2010, 25, 024005. 421

(19) Egard, M.; Johansson, S.; Johansson, a. C.; Persson, K. M.; Dey, 422 a. W.; Borg, B. M.; Thelander, C.; Wernersson, L. E.; Lind, E. Nano 423 Lett. 2010, 10, 809-812.

(20) Johansson, S.; Memisevic, E.; Wernersson, L.-E.; Lind, E. IEEE 425 Electron Device Lett. 2014, 35, 518-520.

(21) Kim, D.; del Alamo, J. IEEE Electron Device Lett. 2010, 31, 806- 427 808.

(22) Fortuna, S. A.j Wen, J, Chun, I S , Li, X Nano Lett. 2008,8, $4421-4427$.

(23) Zhang, X.; Zou, J.; Paladugu, M.; Guo, Y.; Wang, Y.; Kim, Y.; 431 Joyce, H. J.; Gao, Q.; Tan, H. H.; Jagadish, C. Small 2009, 5, 366-369. 432 (24) Fortuna, S.; Li, X. IEEE Electron Device Lett. 2009, 30, 593-595. 433

(25) Dowdy, R.; Walko, D. A.; Fortuna, S. A.; Li, X. IEEE Electron 434 Device Lett. 2012, 33, 522-524.

(26) Miao, X.; Zhang, C.; Li, X. Nano Lett. 2013, 13, 2548-2552. 436 
437 (27) Zhang, C.; Li, X. Solid-State Electron. 2014, 93, 40-42.

438 (28) Zhang, C.; Dowdy, R.; Li, X. Device Research Conference (DRC) 439 2013, 63-64.

440 (29) Dowdy, R.; Walko, D.; Li, X. Nanotechnology 2013, 24, 035304.

441 (30) Borgström, M. T.; Immink, G.; Ketelaars, B.; Algra, R.; Bakkers, 442 E. P. a M. Nat. Nanotechnol. 2007, 2, 541-544.

443 (31) Givargizov, E. J. Cryst. Growth 1975, 31, 20-30.

444 (32) Dayeh, S. A.; Picraux, S. T. Nano Lett. 2010, 10, 4032-4039.

445 (33) Fröberg, L.; Seifert, W.; Johansson, J. Phys. Rev. B 2007, 76, 446153401.

447 (34) Zi, Y.; Jung, K.; Zakharov, D.; Yang, C. Nano Lett. 2013, 13, 448 2786-2791.

449 (35) Persson, A. I.; Larsson, M. W.; Stenström, S.; Ohlsson, B. J.; 450 Samuelson, L.; Wallenberg, L. R. Nat. Mater. 2004, 3, 677-681.

451 (36) Schwarz, K. W.; Tersoff, J. Nano Lett. 2011, 11, 316-320.

452 (37) Schwarz, K. W.; Tersoff, J. Nano Lett. 2012, 12, 1329-1332.

453 (38) Dowdy, R. S.; Zhang, C.; Mohseni, P. K.; Fortuna, S. A.; Wen, 454 J.-G.; Coleman, J. J.; Li, X. Opt. Mater. Express 2013, 3, 1687.

455 (39) Chou, Y.-C.; Hillerich, K.; Tersoff, J.; Reuter, M. C.; Dick, K. a; 456 Ross, F. M. Science 2014, 343, 281-284. 